\title{
Traffic Flow Signal based Traffic Event Reconstruction using Sequential Monte Carlo Methods
}

\author{
Xiangwen Feng ${ }^{1, a}$, Song $\mathrm{Xu}^{2, \mathrm{~b}}$, Xuefeng $\mathrm{Yan}^{1, \mathrm{c}}$ \\ ${ }^{1}$ College of Information and Technology, Nanjing University of Aeronautics and Astronautics, \\ Nanjing, Jiangsu, China \\ ${ }^{2}$ Systems Engineering Research Institute, Beijing, China \\ afxwran@gmail.com, bsonglcl@sohu.com, ${ }^{\mathrm{c} x u e f e n g . y a n @ g m a i l . c o m ~}$
}

Keywords: Traffic Flow Signal, SMC, DDDAS, event reconstruction

\begin{abstract}
According to the nonlinear and non-Gaussian characteristics of the traffic flow, we propose a SMC based traffic flow congestion event reconstruction framework based on traffic flow signals. The simulation states can get close to the real scene continuously along with the data assimilation model assimilates the real-time traffic signals constantly. The congestion event in real scene can be estimated based on the simulation data. Thus, we can estimate the congestion in different particles and finally reconstruct the congestion event. This framework can evaluate the current roads' states based on the reconstruction results, and then the range and the start position of the congestion can be determined. Related experimental results are presented and analyzed.
\end{abstract}

\section{Introduction}

The rapid developments of static and dynamic sensors on real road network make it possible to get real-time traffic signals which can reflect the critical nature of traffic flow. In 2002, Martin Treiber and Dirk Helbing presented an adaptive smoothing algorithm which could get the vehicles' density, the vehicles' average speed quickly and accurately based on fixed sensors [1]. In 2007 Herrera and Bayen introduced many methods to acquire the vehicles' density based on mobile sensors [2]. Obviously, the rational use of real-time traffic signals is the key to improve the accuracy of traffic flow event reconstruction.

Event reconstruction has been widely used in various application domains, including digital investigation [3], digital filtering [4], power systems [5] and microscopic particles [6]. Traditionally, regression, inversion, and optimization are widely used although they have limitations for complex event reconstruction systems. Therefore, many researchers seek other approaches to the solutions. The initial method of Markov Chain Monte Carlo stochastic methodology and the improved methods of SMC methods were adopted to the dynamic data driven event reconstruction for atmospheric releases by Kosovic et al. (2007) [7]. In traffic flow systems, a short-term network traffic prediction method was proposed by Zhang and Huang in [8]. They use particle filter method and the ACD model to deal with the complex traffic signals. Bi Chang and Fan in [9] propose a particle filter based approach to estimate freeway traffic state, through the experiment which used the freeway link between the west of Peace Bridge and the west of San Yuan Bridge of the third ring in Beijing, they proved PF have an encouraging estimation performance.

\section{SMC based Traffic Flow Event Reconstruction}

Traffic Signal Collection using sliding window. Normally, the traffic signals at one time cannot represent the traffic flow appropriately due to the traffic data is changeable and has the feature of mobility. Here we introduce the thought of sliding window to cache and update the traffic signals fleetly, and increase the stability of the sensor data as well as keeping its real-time characteristic. The sliding window is formalized in equation (1). $\mathrm{W}_{T}$ and Length are the total and current size of the 
sliding window respectively, Tail is a pointer that points to the location of a new arrival data, $\mathrm{f}$ is the data update rule.

QueueW $=<\mathrm{W}_{T}$, length,Tail,f $>$

Based on the thought of sliding window, we put forward the traffic signals management model in Fig. 1. The "Traffic Sensors" contains N sensors spread in the real road which can monitor M types of traffic signals. We can collect the sensor data and set up $\mathrm{M}^{*} \mathrm{~N}$ sliding windows using "Sliding Window Generator". "Sliding Window Manager" provides certain real-time data to "Assimilation Model” by dealing with the traffic signals stored in sliding windows.

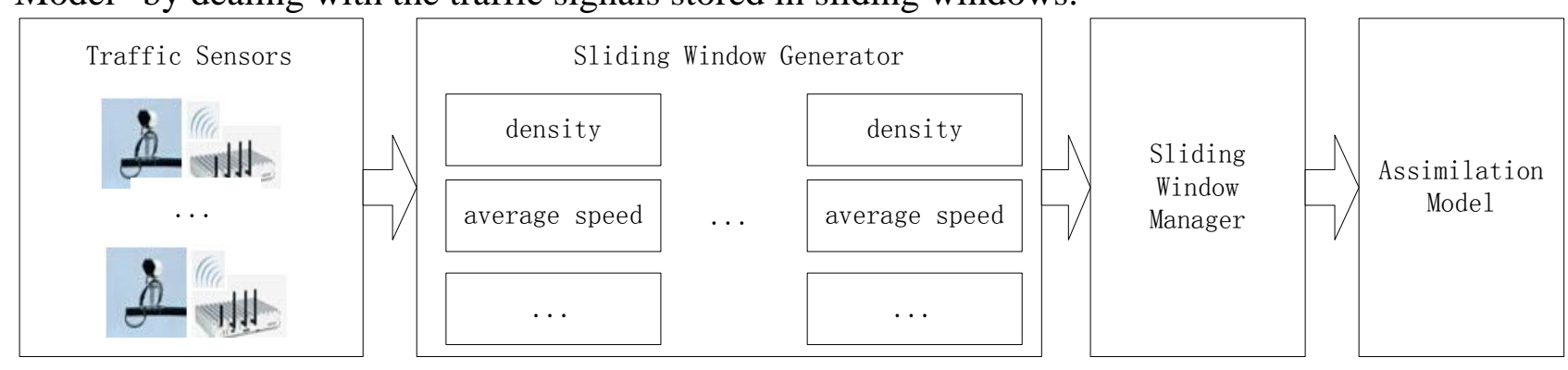

Fig.1 Traffic signals management model based on sliding window

SMC based Traffic Signal Processing in Assimilation Model. SMC has the ability to overcome rapid changes in dynamic systems. Yet, it will undergo particle degeneracy after a few iteration of processing. In this paper, we mainly use two ways to deal with this problem: firstly, we introduce the random model between the resampling step and the next iteration; secondly, strengthen the dynamic relationship between the simulation model, the real-time data and the reconstruction model based on the DDDAS's thoughts. According to the system model and the method mentioned above, The SMC algorithm based traffic signal processing in assimilation model can be summarized into the following four steps. We use step 1 to initialize $\mathrm{N}$ simulation particles, the loop of step 2 and step 3 achieves continuous traffic flow state-space conversion.

Step 1: Initialize N simulation particles. Firstly, get the road types, links, geometric characteristics and the capacity of the road network by Google Map or detecting in real scene; get the type of vehicles and its traffic parameters by official statistics and empirical data. Then, initialize $\mathrm{N}$ simulation particles by specifying the car-following model, lane-changing model et al. Each particle is independent of each other and can be synchronized to simulate the next time's traffic flow states based on the specified traffic scene and travel model.

Step 2: Computing the $\mathrm{N}$ particles' weight by comparing the real-time traffic signals with the simulation data. In this paper, the weight of a particle at time t can be calculated using Equation (2).Where a is the penalty factor that shown in Equation (3), sensor_density ${ }_{[1-k]}(t)$ is the density of traffic flow at time $t$, which is got from the "Sliding window manager" based on k sensors on the road network. sim_density ${ }_{[1-k]}(i, t)$ is the density of the traffic flow that gets from particle $\mathrm{i}$ based on the Measurement Model. Equation (5) shows the computing method of sum. At the beginning of the simulation, we set $b=1$, and based on the simulation results, we can set $b$ as shown in Equation (4). If the value of $a, b$, or sum is 0 , we value it as 0.1 . In order to judge the particles' quality directly, we use the weight normalization algorithm shown in Equation (6) to transform the weight into $(0,1)$.

$$
\begin{aligned}
& \text { weight }(i, t)=1 /(\lceil\mathrm{a}\rceil *\lceil b\rceil *\lceil\operatorname{sum}\rceil) \\
& a=\mid \max \left(\text { sensor_density }_{[1-k]}(t)\right)-\max \left(\operatorname{sim} \_\operatorname{density}_{[1-k]}(i, t)\right) \mid \\
& \mathrm{b}=\mid \max (\text { sensor_aveSpeed } \\
& {[1-k]} \\
& \text { sum }=\sum_{\mathrm{i}=1}^{K} \mid \operatorname{sim} \text { _density }
\end{aligned}
$$




$$
\text { NormWeight(i, t) }=\text { weight }(\mathrm{i}, \mathrm{t}) / \sum_{j=1}^{N} \text { weight }(\mathrm{j}, \mathrm{t})
$$

Step 3: Resample the $\mathrm{N}$ particles based on the weight mentioned above. Here, the resampling algorithm uses the thought of stratified statistics. Firstly we get a subset of the particles. Then calculate the replication sequence which saves the particle number to be copied using certain algorithms. Lastly, update the states of each particles based on the replication sequence. In this paper,

we draw a uniform distribution $u_{i}\left(\frac{1}{n}, \frac{2}{n}, \frac{3}{n} \ldots \frac{n-1}{n}, \frac{n}{n}\right)$ $\sum_{j=1}^{\mathrm{m}-1} \operatorname{NormWeight}(\mathrm{k}, \mathrm{j})<u_{i} \leq \sum_{j=1}^{\mathrm{m}} \operatorname{NormWeight}(\mathrm{k}, \mathrm{j})$, then the m simulation particle will be covered by the i particle. In order to solve the particle degeneracy problem, the proper random factors which have an inverse proportion function against the average weight are added in the vehicles' parameters (position, speed, acceleration, etc.).

After that, In order to speculate whether there is a traffic jam in the real road based on the simulation results, we divide the road into $\mathrm{N}$ road segments. We think the road is smooth when the vehicle density is smaller than smoothDensity, while the road is in a jam when the density is bigger than blockDensity. Assume that the vehicle density of the $\mathrm{N}$ road segments at time $\mathrm{t}$ is $\{$ density[1](t), density[2](t) ... density[N](t)\}. In this paper, we believe that the range of a traffic jam is the longest continuous road segments that are not smooth and exit block segments, and the first road segment of the longest continuous segments is the start scope of the jam.

\section{Experiments and discussion}

Here we apply the event reconstruction model to the real road scene (the main road network from the Ming Palace to the Zhongshan Gate in Nanjing). The road network defined in MovSim is shown in Fig. 2. The $1000 \mathrm{~m}$ road marked in red is the most prone to cause congestion events. The traffic flow congestion event reconstruction experiment is carried out in two steps

Step 1, set the position of the breakdown point at 500m. Run the simulation for 300 time-steps after initializing a single-thread simulation based on the described traffic scene. Record all the vehicle parameters at each time-step as the "real" traffic signals.

Step 2, remove the breakdown point and restart the simulation with 10 simulation particles. The particles will get close to the real scene continuously along with the data assimilation model assimilate the traffic signals constantly. After 180 time-steps of simulation, the state is shown in Fig. 3. We can see that, the simulation model can accurately simulate the real traffic conditions on complex road network. We estimate that there exists a traffic jam between $350 \mathrm{~m}$ and $500 \mathrm{~m}$ because the breakdown of the lane among $350 \mathrm{~m}$ and $400 \mathrm{~m}$. Thus, the traffic flow jam event reconstruction model can estimate the jam event by assimilating real-time traffic signals continuously and reconstruct the congestion event accurately on complex road.

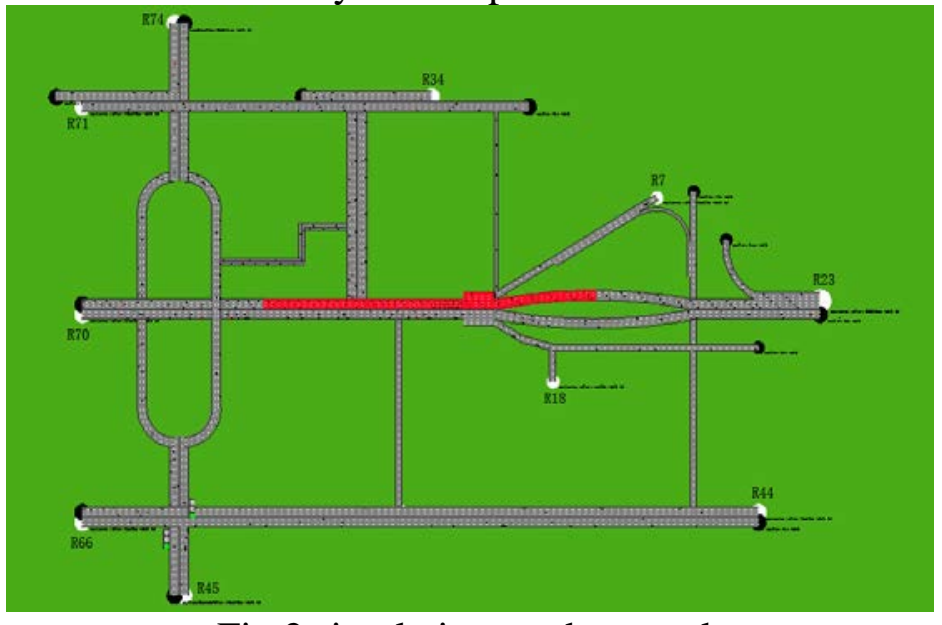

Fig.2 simulation road network 


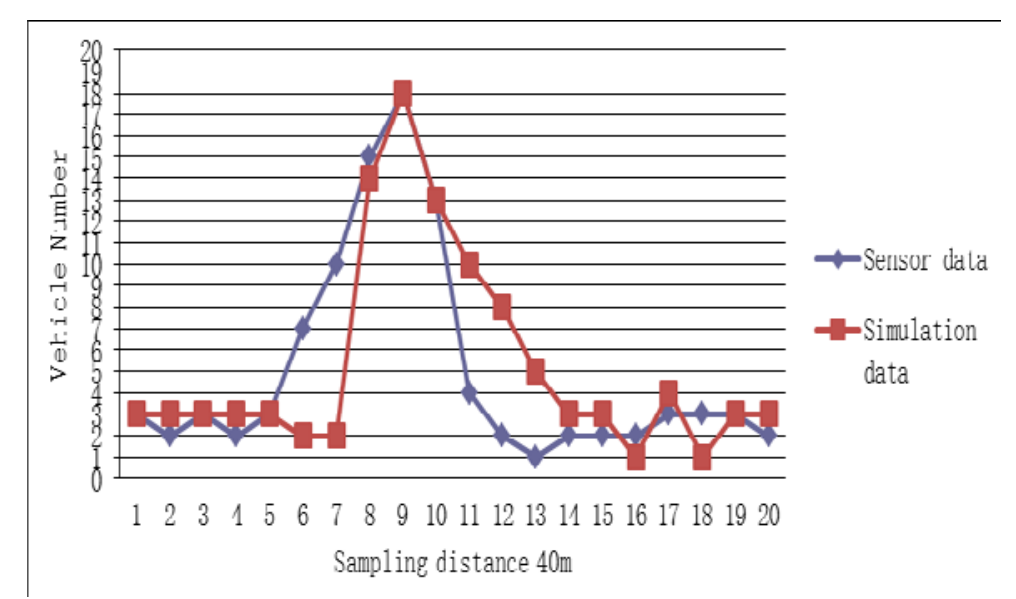

Fig.3 Predicted flow versus observed flow

\section{Acknowledgement}

This work is supported by The Nanjing University of Aeronautics and Astronautics (NS2013091)

\section{Conclusions and future work}

In this paper, the traffic flow signal based traffic event reconstruction is presented, and the traffic flow signal processing using SMC methods is described in detail. The event reconstruction experiment shows that based on the simulation model MovSim, the dynamic data driven event reconstruction system can reconstruct the traffic congestion event accurately with the proper use of real-time traffic signals.

\section{References}

[1] Treiber M, Helbing D. Reconstructing the spatio-temporal traffic dynamics from stationary detector data[J]. Cooperative Transportation Dynamics, 2002, 1(3): 3.1-3.21.

[2] Herrera J C, Bayen A M. Traffic flow reconstruction using mobile sensors and loop detector data[J]. 2007.

[3] Gladyshev P. Formalising event reconstruction in digital investigations[D]. University College Dublin, 2004.

[4] Ayodeji O O, Jiya J Y, Hale J M. Event Reconstruction by Digital Filtering[J]. 2013.

[5] Bill Brown P E, Kozlowski M, Center S D C P C. Power System Event Reconstruction Technologies for Modern Data Centers[J]. 2006.

[6] Spataro S. Simulation and event reconstruction inside the PandaRoot framework[C]//Journal of Physics: Conference Series. IOP Publishing, 2008, 119(3): 032035.

[7] Serban G S, Aines R. Dynamic data-driven event reconstruction for atmospheric releases[J]. 2007.

[8] Zhang G, Huang D. Short-Term Network Traffic Prediction with ACD and Particle Filter[C]//Intelligent Networking and Collaborative Systems (INCoS), 2013 5th International Conference on. IEEE, 2013: 189-191.

[9] Bi J, Chang C, Fan Y. Particle Filter for Estimating Freeway Traffic State in Beijing[J]. Mathematical Problems in Engineering, 2013. 